\title{
Bellini's collecting duct renal carcinoma: report of two cases and review of the literature
}

\section{Carcinoma renal de ductos colectores de Bellini: presentación de dos casos y revisión de la literatura}

(DGarazi Gutiérrez-Zurimendi, ${ }^{1 *}$ (D) Gonzalo García de Casasola-Rodríguez, ${ }^{1}$ (D) Ibon Urrestizala-Peña, ${ }^{2}$

(10) Mikel Aizcorbe-Gómez, ${ }^{1}$ Asier Maldonado-De Sasia, ${ }^{1}$ Aranzazu Urresola-Olabarrieta. ${ }^{1}$

Keywords:

Bellini's collecting duct renal carcinoma, renal cancer, urothelial cancer, nephrectomy, chemotherapy

Autor para correspondencia:

*Garazi Gutiérrez-Zurimendi. Hospital

Universitario

Cruces-Gurutzeta.

Plaza de Cruces, S/N 48903 Barakaldo, Bizkaia, España. Correo electrónico: garazigz@ gmail.com

\section{Abstract}

Clinical cases: We present two cases of Bellini's Collecting Duct Renal Carcinoma (CDRC) that appear as suspicious kidney masses with enhancement of the urotelium, without a determinant radiological appearance, diagnosed by anatomo-pathological analysis. We revise the medical history, histological findings and make a review of the literature.

Importance / Clinical implication: Bellini's collecting duct renal carcinoma is a very rare and aggressive type of kidney cancer (less than 1\%), usually diagnosed at advanced stages. It does not present specific radiological characteristics, which makes necessary the histologic and inmunohistochemical analysis to confirm the diagnosis. We must suspect CDRC in infiltrative medular renal masses that do not affect kidney shape, and treat as soon as possible performing surgery, whenever feasible, normally associated with adjuvant chemotherapy. Nevertheless, it usually has an unfavourable evolution.

Conclusion: It is imperative to diagnose and treat this kind of tumours as soon as possible due to its aggressiveness and fatal prognosis.

Citación: Gutiérrez-Zurimendi G., García de Casasola-Rodríguez G., Urrestizala-Peña I., Aizcorbe-Gómez M., Maldonado-De Sasia A., Urresola-Olabarrieta A. Carcinoma renal de ductos colectores de Bellini: presentación de dos casos y revisión de la literatura. Rev Mex Urol. 2022;82(1):pp 1-10

\footnotetext{
${ }^{1}$ Hospital Universitario Cruces, Barakaldo, Bizkaia. España

${ }^{2}$ Hospital Universitario Galdakao, Galdakao, Bizkaia. España
}

Recibido: 07 de diciembre de 2020. Aceptado: 23 diciembre de 2021

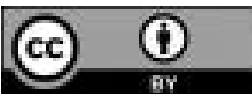




\section{Carcinoma renal de ductos colectores de Bellini: presentación de dos casos... Gutiérrez-Zurimendi G., et al.}

Palabras clave: carcinoma renal de los ductos colectores de Bellini, cáncer renal, tumor urotelial, nefrectomía, quimioterapia

\section{Resumen}

Descripción de casos clínicos: Presentamos dos casos de carcinoma renal de ductos colectores, que se manifiestan como masas renales sospechosas de malignidad con realce del urotelio sin una apariencia radiológica determinante, diagnosticados a partir del análisis anatomo-patológico de la muestra. Correlacionamos la historia clínica con los hallazgos histológicos y realizamos revisión de la literatura.

Relevancia / Implicación clínica: El carcinoma renal de ductos colectores de Bellini (CRDC) es una variante muy infrecuente y agresiva de tumor renal (menos del 1\%) que suele diagnosticarse en estadios avanzados. El diagnóstico diferencial radiológico con otros tipos de masas renales no es sencillo, ya que no hay características patognomónicas que lo definan, por lo que ha de sospecharse ante lesiones renales de predominio medular con crecimiento infiltrativo que no deformen la silueta renal, y tratarlo lo antes posible mediante exéresis quirúrgica siempre que sea factible, en ocasiones asociada a quimioterapia adyuvante, aunque suele presentar una evolución desfavorable.

Conclusión: Debido a su agresivo comportamiento, son imperativos un diagnóstico y tratamiento precoces, aunque pese a ello presenta un pronóstico infausto.

\section{Introducción}

Los tumores renales representan aproximadamente el $2-3 \%$ de las neoplasias en adultos. ${ }^{(1)}$ Según la American Cancer Society, en Estados Unidos en 2021 la incidencia global de cáncer renal y de pelvis renal fue de 76080 nuevos casos de cáncer de riñón (www.cancer.org). ${ }^{(1)}$ El carcinoma renal de los ductos colectores de Bellini (CRDC) es una variante histológica muy infrecuente de tumor renal, representando menos del $1 \%$ de los tumores renales. ${ }^{(2,3)}$

Puede aparecer a cualquier edad (según la OMS, aparece entre los 8 y 83 años), ${ }^{(2-4)}$ principalmente en el sexo masculino $(2: 1){ }^{(2)}$
Habitualmente se diagnostica en estadios avanzados de la enfermedad, pudiendo ser metastásicos al diagnóstico hasta en el 33-50\% de los casos, ${ }^{(2-5)}$ por lo que la mayoría de los pacientes presentan un pronóstico infausto.

Las pruebas de imagen abdominal, como la tomografía axial computarizada (TAC) o la resonancia magnética $(\mathrm{RM})$, no permiten diferenciar este tipo de tumor renal de otras variantes ya que, aunque suelen mostrar unas características típicas, no existen rasgos patognomónicos que lo definan. Por tanto, para su diagnóstico definitivo es indispensable el análi- 
sis anatomo-patológico e inmunohistoquímico de la muestra. ${ }^{(5-7)}$

El tratamiento está basado en la resección quirúrgica de la lesión siempre que sea posible, ya que parece ser el único tratamiento potencialmente curativo en pacientes con tumor localizado. $^{(6-10)}$ Se han probado tratamientos sistémicos como quimioterapia, antiangiogénicos o inmunoterapia, sin haberse objetivado gran respuesta a los mismos. Aunque hay pocos casos reportados, los regímenes de quimioterapia similares a los utilizados para neoplasias de urotelio son los más empleados debido a la similitud histológica y etiopatogénica de este tipo de tumor con los de origen urotelial. ${ }^{(2,7-9)}$

A continuación, presentamos 2 casos de tumor renal de los ductos colectores de Bellini diagnosticados en nuestro servicio de salud, atendiendo a su diagnóstico, tratamiento y evolución.

\section{Caso clínico 1}

Paciente varón de 82 años, con antecedentes de hipertensión arterial, fibrilación auricular anticoagulada con dabigatrán y gastroduodenitis erosiva, que en estudio por elevación de tran- saminasas es diagnosticado de masa renal derecha de aproximadamente $7 \mathrm{~cm}$ y engrosamiento y realce pielocalicial heterogéneo en riñón izquierdo, con sospecha de neoplasia urotelial. El paciente niega dolor, y refiere que ocasionalmente ha presentado episodios de hematuria leve autolimitada. Por este motivo, se realizan citologías, con resultado negativo, y cistoscopia con hallazgo de varias zonas eritematosas milimétricas, no sospechosas de malignidad. Se decide inclusión en lista quirúrgica para realización de ureterorrenoscopia (URS) izquierda diagnóstica y cistoscopia intraquirófano con toma de biopsias múltiples randomizadas de vejiga. Se realiza intervención, sin hallazgos sospechosos a nivel macroscópico, y con anatomía patológica que descarta afectación tumoral urotelial.

El TAC describe la lesión como una masa renal derecha exofítica, heterogénea, con alguna calcificación central, que contacta con el seno renal obliterando parcialmente el cáliz superior. Asimismo, parece que contacta con la superficie posterior del hígado, por lo que no se puede descartar su infiltración. No se objetivan adenopatías locorregionales ni lesiones a distancia (Figura 1). 
Figura 1. Imágenes de TAC
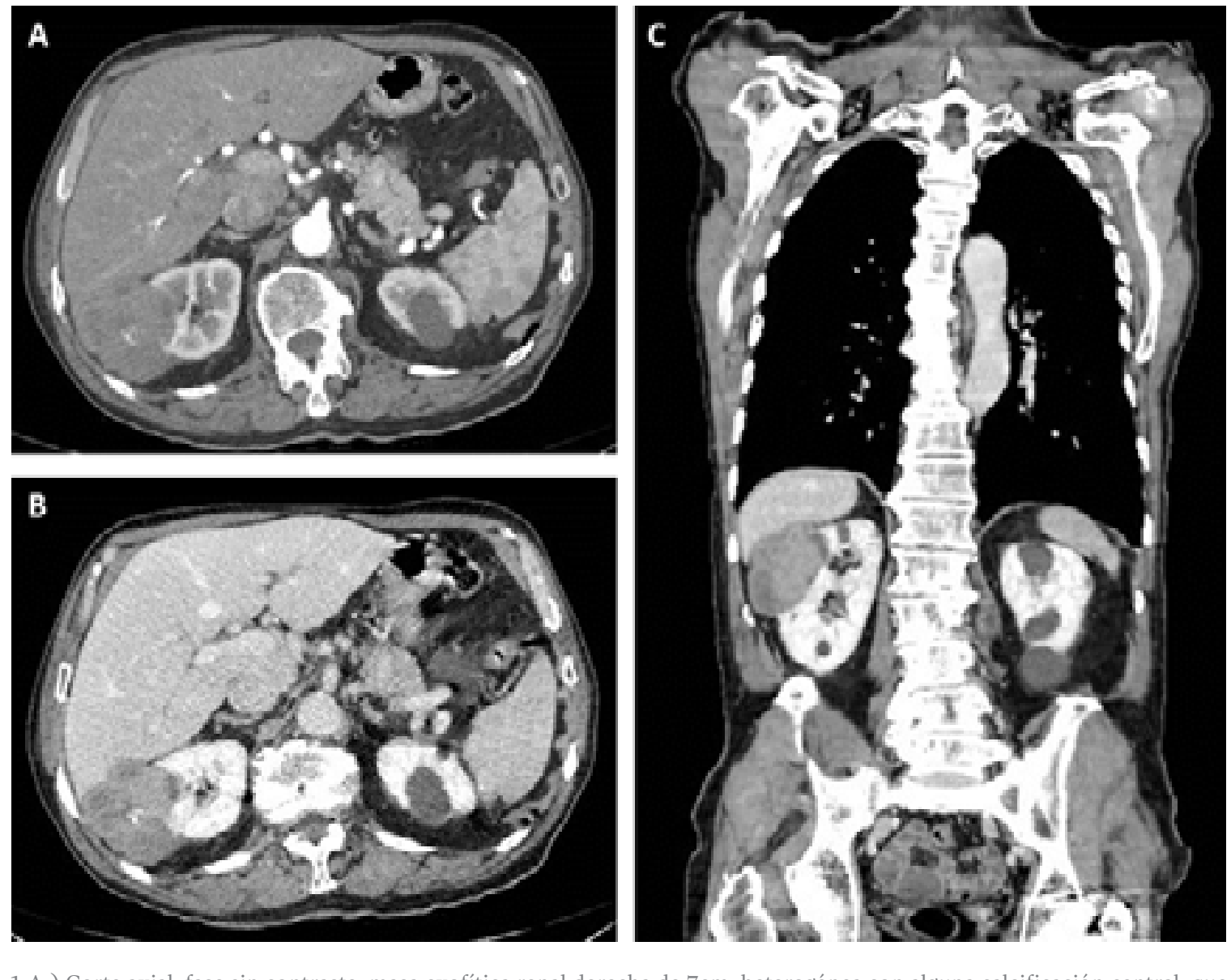

1.A.) Corte axial, fase sin contraste: masa exofítica renal derecha de $7 \mathrm{~cm}$, heterogénea con alguna calcificación central, que contacta con el seno renal obliterando un cáliz superior, y posteriormente con el hígado, sin poderse descartar infiltración. 1.B.) Misma imagen tras administración de contraste: captación simétrica de contraste por ambos riñones. Leve realce de masa descrita. 1.C.) Corte coronal tras administración de contraste: masa renal que contacta con el hígado.

Por tanto, se realiza nefrectomía radical derecha laparoscópica, sin incidencias intraoperatorias. Macroscópicamente se observa un tumor exofítico en cara lateral del riñón derecho, a nivel interpolar. El paciente evoluciona favorablemente durante el postoperatorio, siendo dado de alta al segundo día.

La anatomía patológica de la pieza quirúrgica muestra una tumoración de crecimiento nodular y patrón arquitectural heterogéneo, papilar, tubulopapilar y ductal infiltrante, con acusada desmoplasia del estroma y con atipia citológica con nucléolos prominentes. Se observan amplias zonas de hemorragia y necrosis. La tumoración crece de manera expansiva a la grasa perirrenal, quedando en contacto con la fascia sin sobrepasarla. Con técnicas de inmunohistoquímica se observa positividad para PAX8, INI-1, CK19, y focal para CK7, 34 beta E12 y anhidrasa carbónica, siendo negativa para p63, lo que va a favor de que se trate de un carcinoma del ducto colector, de $8.5 \mathrm{~cm}$, que infiltra el tejido adiposo perirrenal (pT3aN0M0), con margen quirúrgico libre (Figura 2). 
Figura 2. Imágenes de anatomía patológica a nivel microscópico

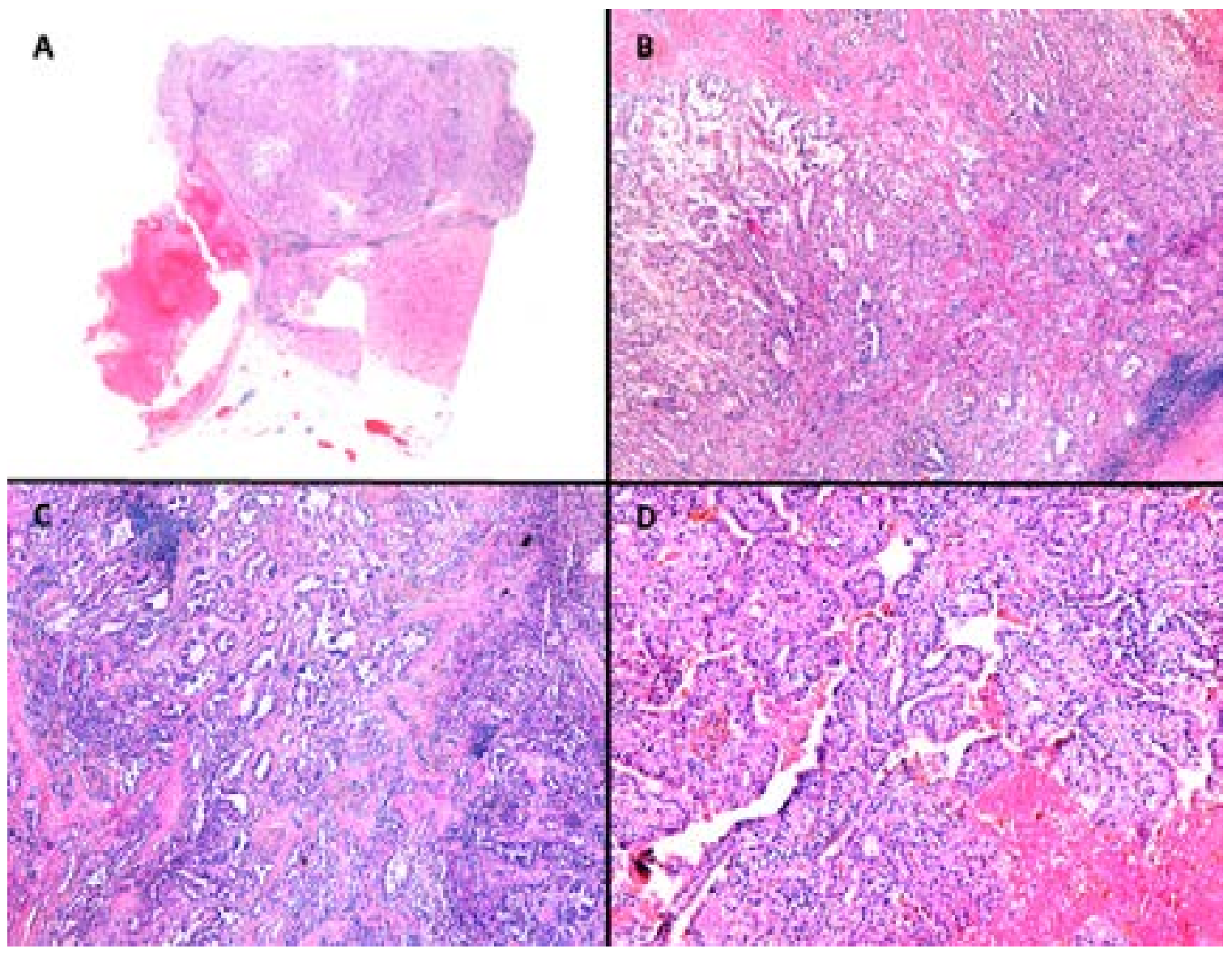

2.A.) Tumoración multinodular parcialmente rodeada por pseudocápsula con componente hemorrágico quístico, con inflamación crónica en los límites. 2.B.) La lesión crece de manera infiltrativa y presenta túbulos con estroma desmoplásico, así como crecimiento papilar. 2.C.) Patrón de crecimiento acinar con estroma desmoplásico. 2.D.) Patrón papilar con espacios quísticos en parte central.

Al mes de la cirugía, el paciente se encuentra subjetivamente muy bien, con función renal conservada. Se realiza nuevo control a los 3 meses de la cirugía y a los 9 meses, sin objetivarse recidiva local ni a distancia. El paciente continúa vivo hoy en día, tras 11 meses de la cirugía, con buen control de la enfermedad.

\section{Caso clínico 2}

Mujer de 54 años, sin antecedentes médico-quirúrgicos de interés, que a raíz de presentar hematuria se somete al estudio pertinente, sin detectar lesiones intravesicales ni alteraciones citológicas. Se realiza TAC con contraste con hallazgo de masa renal de aproximadamente $3 \mathrm{~cm}$ en polo superior izquierdo, sospechosa de malignidad; no obstante, también se observa un realce del urotelio del 
sistema pielocalicial de este riñón, por lo que el radiólogo plantea un posible diagnóstico diferencial con una pielonefritis aguda (Figura 3).

Figura 3. Imágenes de TAC

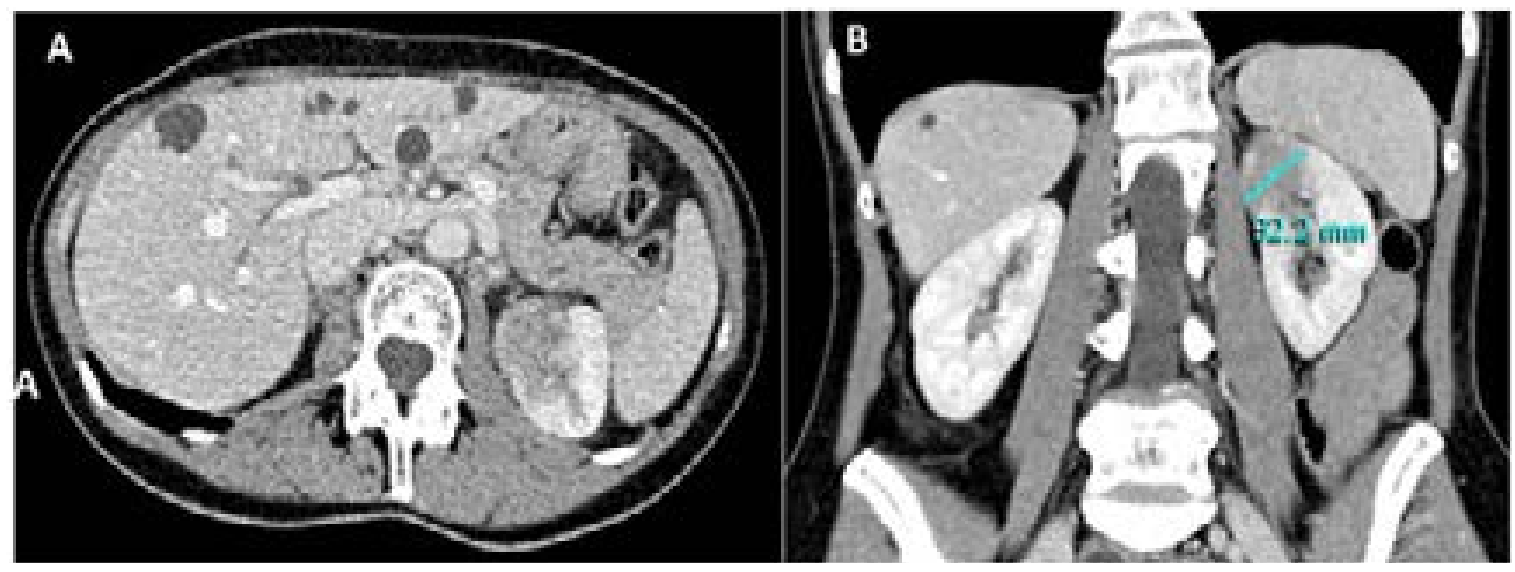

3.A.) Corte axial, fase con contraste: Masa de $3,2 \mathrm{~cm}$ en polo renal superior izquierdo sospechosa de malignidad, con realce del urotelio del sistema pielocalicial. 3.B.) Corte coronal, fase con contraste: lesión previamente descrita visualizada en plano coronal. Relación con estructuras adyacentes.

Ante estos hallazgos, se revisa la historia clínica y las imágenes, con alta sospecha de tratarse de una lesión tumoral del parénquima renal. Se realiza estudio de extensión, sin objetivarse adenopatías ni lesiones a distancia. Aunque la primera sospecha es que se trate de una lesión parenquimatosa, teniendo en cuenta el realce del sistema pielocalicial y que en imágenes parece estar en contacto con la vía urinaria, se presenta el caso en sesión y se decide realizar nefrectomía radical laparoscópica con margen ureteral amplio. La cirugía transcurre sin incidencias y la evolución postoperatoria es favorable, siendo dada de alta en el segundo día postoperatorio.

El análisis anatomopatológico muestra una lesión blanquecino-amarillenta excrecente de aspecto verrucoso, de $0.6 \mathrm{~cm}$ de diámetro, que se continúa con una tumoración parenquimatosa, córtico-medular, mal delimitada, de $3 \mathrm{~cm}$. Microscópicamente, se observa una prolifera- ción de crecimiento túbulo papilar con atipia, anaplasia y frecuentes figuras de mitosis, localizada en médula que infiltra focalmente la pelvis renal y se extiende hasta corteza respetando los glomérulos. Las técnicas de inmunohistoquímica muestran positividad de CK-19, vimentina, Ulex europeus y 34 Beta E-12, siendo negativas p63 y CD-10.

Por tanto, se confirma el diagnóstico de carcinoma de los ductos colectores de Bellini de $3 \mathrm{~cm}$, que infiltra focalmente la pelvis renal, respetando el resto de las estructuras hiliares y el borde ureteral, pT1a N0 M0.

Se realiza control a los 3 meses, sin datos de enfermedad a distancia. A los 6 meses, el TAC revela progresión tumoral, objetivándose una masa retroperitoneal izquierda en lecho de nefrectomía y metástasis hepáticas y óseas. Se realiza biopsia de las lesiones hepáticas, siendo positiva para infiltración por carcinoma de los ductos colectores de Bellini, por lo que se 
inicia quimioterapia paliativa con Carboplatino-Gemcitabina. Tras tres ciclos se objetiva una respuesta parcial de las lesiones, y tras seis ciclos la enfermedad permanece estable. Finaliza el tratamiento a los 6 meses, pero, poco después, la paciente empeora clínicamente debido a la progresión de la enfermedad, por lo que es ingresada en oncología médica, descartándose tratamiento activo ante mal estado general y mal pronóstico. Fallece dos semanas después de su ingreso, con una supervivencia de 20 meses desde su diagnóstico.

\section{Discusión}

El carcinoma renal de los ductos colectores de Bellini es, como hemos comentado, una de las variantes de tumor renal más infrecuentes, más frecuente en el sexo masculino (relación $2: 1) .^{(2,8,11)}$ y con una media de edad de presentación de en torno a 55 años (8-83 años). ${ }^{(2-4,11)}$

La mayoría de las neoplasias renales se forman en células del túbulo contorneado proximal, como los carcinomas de células claras, ${ }^{(6,10-12)}$ mientras que otros tipos más infrecuentes se originan en el epitelio del túbulo contorneado distal, ${ }^{(2,11)}$ como los oncocitomas, los cromófobos y los CRDC de Bellini. ${ }^{(6)}$

Desde el punto de vista embriológico, los túbulos colectores, cálices, pelvis renal y uréter tienen un origen común a partir de la yema ureteral del conducto de Wolff, ${ }^{(11-13)}$ lo que explicaría la asociación ocasional de estos tumores con el carcinoma urotelial transicional. En cuanto a su histoquímica, tanto los tumores de los ductos colectores como los uroteliales pueden presentar positividad a la mucina (a diferencia de otros tumores del parénquima renal como el de células claras). ${ }^{(6,7,10,11)}$
En uno de los casos presentados, el paciente fue sometido a ureterorrenoscopia diagnóstica por imagen de aspecto sospechoso en la pelvis renal del riñón contralateral, siendo su análisis negativo para malignidad. No obstante, hay que tener presente la posible asociación de ambas entidades. ${ }^{(11)}$

Su presentación clínica es similar a la del resto de tumores renales, siendo asintomático en estadios iniciales, por lo que suele ser un hallazgo casual en pacientes que consultan por otros motivos. Los síntomas suelen aparecer en un estadio muy avanzado, cuando el tumor invade la cápsula renal y produce dolor lumbar, masa abdominal palpable y/o hematuria, acompañándose en ocasiones de síndrome general. ${ }^{(2,6,11)}$ En el $30-50 \%$ de los casos puede ser metastásico al diagnóstico, ${ }^{(2-5)}$ siendo los principales órganos diana el hueso, hígado y pulmón. ${ }^{(12)}$

$\mathrm{Su}$ diagnóstico es difícil sobre todo por su baja incidencia, por lo que debe realizarse diagnóstico diferencial con otros tumores renales: ${ }^{(12)}$ carcinoma papilar, ${ }^{(2,10)}$ carcinoma urotelial con diferenciación glandular, ${ }^{(2,10)}$ carcinoma metastásico del tracto gastrointestinal y pulmón, ${ }^{(2,10)}$ y carcinoma medular renal. ${ }^{(10)}$

Los hallazgos radiológicos muestran lesiones heterogéneas, normalmente de localización central (predominio medular en la vecindad del sistema excretor), con preservación del contorno renal, hipovasculares y con mínimo realce con contraste (a diferencia del cáncer de células claras, donde el realce es más intenso), en ocasiones con componente quístico y crecimiento infiltrativo afectando al seno renal. $(5,11,12)$ Pero estos hallazgos no son específicos, por lo que hay que realizar diagnóstico diferencial con otras variantes de tumor renal y es necesario el diagnóstico de confirmación mediante su análisis anatomo-patológico. ${ }^{(5-7)}$. 
Macroscópicamente, el CRDC suele presentarse como una masa de gran tamaño (2.5 a $12 \mathrm{~cm}$ ), de localización medular o piélica, que infiltra secundariamente la corteza renal y puede invadir el tejido adiposo del seno renal y perirrenal. ${ }^{(2,11)}$

Microscópicamente, presenta un patrón de crecimiento formado por túbulos y papilas dilatadas que infiltran el parénquima renal y pueden generar un aspecto quístico "en esponja”. ${ }^{(11,12)}$ Aparecen glándulas irregulares y un estroma desmoplásico con infiltrado inflamatorio acompañante, en ocasiones con intensa formación de tejido fibrótico secundario. Las células son pleomórficas, con un alto grado nuclear y nucléolos prominentes, con morfología "en tachuela". ${ }^{7,10,13)}$ Fleming et al. describieron los cambios de hiperplasia del epitelio de los conductos colectores en la médula renal adyacente al tumor como un rasgo típico de estas lesiones, que refrenda su origen en los túbulos colectores. ${ }^{(6)}$

Las técnicas de inmunohistoquímica han permitido llegar al diagnóstico definitivo de este tipo de lesiones. Aunque puede ser variable, la inmunohistoquímica habitualmente es positiva para citoqueratinas de alto peso molecular (CK19, CK34ßE12, CK7, CK8/18...), lo que lo diferencia del cáncer renal de células claras, ${ }^{(14)}$ así como Fez 1, Ulex-Europeus, mucina, lisozima, lecitinas/peanut aglutinina, ${ }^{(2,6,7,11)}$ vimentina, PAX2 y PAX8, ${ }^{(14)}$ y negativa para p63 (a diferencia del origen urotelial, PAX8(-)/ p63(+)),,$^{(14)}$ y otros marcadores del túbulo contorneado proximal. En cuanto a la citogenética de estos tumores, la patogénesis molecular no está clara debido al pequeño número de casos descritos, aunque se han descrito monosomías en los cromosomas $1,6,14,15$ y $22 .^{(10-14)}$
Analizando los casos publicados, los datos sobre supervivencia son dispares ya que se trata de pequeñas series, y dependen del estadio de la enfermedad al diagnóstico. En general, la mayoría coinciden en que alrededor del 33-50\% de los pacientes presenta metástasis al diagnóstico, ${ }^{(2,5,11)}$ y aproximadamente $2 / 3$ de los pacientes fallecen en los primeros 2 años, ${ }^{(2,5,8)}$ lo que refleja su desfavorable pronóstico.

Hoy en día no hay un tratamiento estándar para este tipo de neoplasia. ${ }^{(5,8)}$ El tratamiento está basado en la resección quirúrgica de la lesión siempre que sea posible, ya que parece ser el único tratamiento potencialmente curativo en pacientes con tumor localizado, ${ }^{(7)} \mathrm{o}$ incluso en enfermedad avanzada con intención citorreductora o paliativa, aunque no hay evidencia que demuestre una mejoría de la supervivencia por sí sola en los pocos casos descritos en la literatura. ${ }^{(9)}$

Debido a su habitual presentación en estadios avanzados, así como su tendencia a progresar, en la mayoría de los casos es necesario administrar un tratamiento sistémico, habiéndose probado diversas opciones, como radioterapia, quimioterapia, antiangiogénicos, inmunoterapia, y hasta inhibidores de tirosina kinasa (TKI) (sunitinib o sorafenib), ${ }^{(8)}$ sin haberse objetivado gran respuesta a los mismos. Aunque no existen regímenes claramente validados, parece que las pautas de quimioterapia utilizadas para el tratamiento del carcinoma transicional infiltrante, como Gemcitabina-Cisplatino o carboplatino, ${ }^{(2,5,8,9)}$ en algunos casos han logrado la estabilización o remisión parcial de la enfermedad, debido a la similitud histológica y etiopatogénica de este tipo de tumor con los tumores de origen urotelial, aunque los resultados descritos en la literatura son dispares. ${ }^{(13)}$ 
Carcinoma renal de ductos colectores de Bellini: presentación de dos casos... Gutiérrez-Zurimendi G., et al.

Xiaoyuan Qian et al presentaron una serie de 12 pacientes con carcinoma renal de ductos colectores de Bellini tratados mediante cirugía y/o terapia sistémica, de los cuales 11 pacientes presentaron un tiempo medio de supervivencia de 11 meses y solo 1 paciente sobrevivió más de 5 años tras el diagnóstico. En series previas se habían conseguido resultados similares tras tratamiento quirúrgico y/o sistémico. ${ }^{(15)}$

Por tanto, parece que ni la cirugía ni la quimioterapia han demostrado controlar completamente la progresión de la enfermedad, habiéndose descrito una significativa incidencia de recurrencia local y metástasis a distancia ${ }^{(8)}$ lo que demuestra que se trata de un tumor con un comportamiento muy agresivo, cuyo pronóstico suele ser infausto. ${ }^{(2,11,13)}$

\section{Conclusiones}

El cáncer renal de los ductos colectores de Bellini es una variante infrecuente de tumor renal que ha de sospecharse ante lesiones de predominio medular con crecimiento infiltrativo pero que no deforman la silueta renal, aunque radiológicamente no tiene características definitorias típicas y es necesario su análisis histológico e inmunohistoquímico para confirmar el diagnóstico. Debido a su agresivo comportamiento, es imperativo realizar un diagnóstico y tratamiento precoces, ya que entre un tercio y la mitad de los casos se presenta con metástasis al diagnóstico, lo que empeora sustancialmente el pronóstico. Aunque hay pocos casos descritos en la literatura como para sacar conclusiones robustas, parece que el mejor tratamiento disponible hoy en día se basa en la resección quirúrgica siempre que sea posible, habitualmente acompañada de quimioterapia sistémica con el mismo esquema que los tumores de urotelio en casos de enfermedad a distancia. No obstante, en la mayoría de los casos, el pronóstico suele ser infausto a pesar del tratamiento instaurado.

\section{Taxonomía CRedit}

1. Garazi Gutiérrez-Zurimendi: Conceptualization, Investigation, Project administration, Writing

2. Gonzalo García de Casasola-Rodríguez: Conceptualization, Visualization, Writing

3. Ibon Urrestizala-Peña: Investigation

4. Mikel Aizcorbe-Gómez: Resources

5. Asier Maldonado-De Sasia: Resources

6. Aranzazu Urresola-Olabarrieta: Resources, Supervision, Project Administration

\section{Financiamiento y conflicto de intereses}

Se hace constar la cesión de derechos de copyright a la Revista Mexicana de Urología, así como la declaración de estar libres de cualquier asociación personal o comercial que pueda suponer un conflicto de intereses en conexión con el artículo remitido, y haber respetado los principios éticos de investigación.

Asimismo, se hace constar que no se ha recibido financiación de ningún tipo para la realización del trabajo.

\section{Referencias}

1. Ameican Cancer Society. Key Statistics About Kidney Cancer. Cancer. 2022. [accessed 1 Feb 2022] Available from: https://www.cancer.org/ cancer/kidney-cancer/about/key-statistics.html 
2. Gutiérrez-Domingo A, Gutiérrez-Domingo I, Gallardo-Rodríguez K, Rodríguez-Caulo A. Carcinoma de los conductos colectores de Bellini: una alteración poco frecuente y de mal pronóstico. Rev Mex Urol. 2017 May 26;77(3). [accessed 1 Feb 2022] Available from: https:// revistamexicanadeurologia.org.mx/index.php/ $\mathrm{rmu} /$ article/view/34

3. García-Fadrique G, Ramírez-Backhaus $M$, Morales G, Pontones JL, Jiménez-Cruz JF. Carcinoma de los conductos colectores de bellini. Presentación de un caso y revisión de la literatura. Actas Urol Esp. 2010 Jul;34(7):63941. doi: http://dx.doi.org/10.1016/j. acuro.2010.01.024

4. Craver RD, Correa H, Crapanzano JP, Kumar SR, Gardner RV. Renal collecting duct carcinoma in an 8-year-old child. Pediatr Nephrol Berl Ger. 1996 Feb;10(1):29-32. doi: https://doi. org/10.1007/bf00863434

5. Mishra AK, Manikandan R, Dorairajan LN, Mittal JK, Rekha JS. Bellini Duct Carcinoma: A Rare Entity. J Clin Diagn Res JCDR. 2016 Oct;10(10):PD01-2. doi: https://dx.doi.org/10 .7860\%2FJCDR\%2F2016\%2F21335.8613

6. Vecchione A, Galetti TP, Gardiman M, Ishii H, Giarnieri E, Pagano F, et al. Collecting duct carcinoma of the kidney: an immunohistochemical study of 11 cases. BMC Urol. 2004 Sep 9;4(1):11. doi: https://doi. org/10.1186/1471-2490-4-11

7. Husillos A, Herranz-Amo F, Subirá D, Lledó E, Molina-Escudero R, Hernández-Fernández C. [Collecting duct renal cell carcinoma]. Actas Urol Esp. 2011 Jun;35(6):368-71. doi: https:// doi.org/10.1016/j.acuro.2011.01.012

8. Hu YU, Zhou H, Wang G, Song Z, Zhao C, Wang Y. Collecting duct carcinoma of the kidney: A case report. Oncol Lett. 2015 Jun;9(6):2902-4. doi: https://doi.org/10.3892/ol.2015.3085
9. Méjean A, Rouprêt $\mathbf{M}$, Larousserie $\mathbf{F}$, Hopirtean V, Thiounn N, Dufour B. Is there a place for radical nephrectomy in the presence of metastatic collecting duct (Bellini) carcinoma? J Urol. 2003 Apr;169(4):1287-90. doi: https:// doi.org/10.1097/01.ju.0000050221.51509.f5

10. Díaz Pérez JA, Zamora CJ, García Vera JA, Melo Uribe MA, García Ayala E. [Bellini collecting duct renal carcinoma: case report]. Arch Esp Urol. 2008 Feb;61(1):67-71. doi: https://doi. org/10.4321/s0004-06142008000100010

11. Corbetta J, Sívori F, Ale R, Verdinelli J, González O, Besuchio S, et al. Carcinoma de los túbulos colectores de Bellini. Rev Argent Urol. 2001;66(4):173-82.

12. San Miguel Fraile P, Dos Santos JE, Delgado C, Zungri Telo E, Alvarez C, Iglesias B. [Low grade collecting duct carcinoma of the kidney. Presentation of one case and literature revision]. Actas Urol Esp. 2004 Jun;28(6):47883. doi: https://doi.org/10.1016/s02104806(04)73115-0

13. Uruñuela FL, Calvo JJ, Garibay AMSG de, Aristu JIJ, Paul MAP, Troyas RG, et al. Carcinoma de ductos colectores de Bellini. Arch Esp Urol. 2004;57(2):153-6.

14. Ozcan A, Krishnan B, Truong L. Renal Tumors. In: McManus LM, Mitchell RN, editors. Pathobiology of Human Disease. San Diego: Academic Press; 2014. p. 2869-99. [accessed 1 Feb 2022] Available from: https://doi. org/10.1016/B978-0-12-386456-7.05415-0

15. Qian X, Wang Z, Zhang J, Wang Q, Zhou P, Wang S, et al. Clinical Features and Prognostic Outcome of Renal Collecting Duct Carcinoma: 12 Cases from a Single Institution. Cancer Manag Res. 2020;12:3589-95. doi: https://doi. org/10.2147/CMAR.S244094 